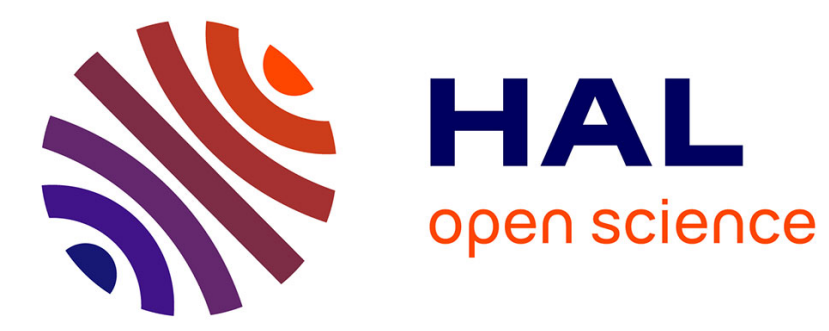

\title{
Crystalline structure's influence on the near-field optical properties of single plasmonic nanowires
}

\author{
Thierry Laroche, Alexandre Vial, M. Roussey
}

\section{To cite this version:}

Thierry Laroche, Alexandre Vial, M. Roussey. Crystalline structure's influence on the near-field optical properties of single plasmonic nanowires. Applied Physics Letters, 2007, 91 (12), pp.123101. $10.1063 / 1.2784389$. hal-00272737

\section{HAL Id: hal-00272737 \\ https://hal.science/hal-00272737}

Submitted on 23 Apr 2021

HAL is a multi-disciplinary open access archive for the deposit and dissemination of scientific research documents, whether they are published or not. The documents may come from teaching and research institutions in France or abroad, or from public or private research centers.
L'archive ouverte pluridisciplinaire HAL, est destinée au dépôt et à la diffusion de documents scientifiques de niveau recherche, publiés ou non, émanant des établissements d'enseignement et de recherche français ou étrangers, des laboratoires publics ou privés. 


\title{
Crystalline structure's influence on the near-field optical properties of single plasmonic nanowires
}

\author{
Thierry Laroche ${ }^{\text {a) }}$ and Alexandre Vial ${ }^{\text {b) }}$ \\ Laboratoire de Nanotechnologie et d'Instrumentation Optique, Institut Charles Delaunay, Université de \\ technologie de Troyes, CNRS FRE 2848, 12, rue Marie Curie BP-2060, F-10010 Troyes Cedex, \\ France \\ Matthieu Roussey ${ }^{\text {c) }}$ \\ Institut FEMTO-ST, Université de Franche-Comté, UMR CNRS 6174, 25030 Besançon, France
}

(Received 7 June 2007; accepted 31 July 2007; published online 17 September 2007)

\begin{abstract}
The finite difference time domain method is employed to study the crystalline structure's influence on the propagation of a local excitation along metallic nanowires of subwavelength cross section. The metallic nanowires are elongated cylinders deposited on a transparent substrate. A tightly focused gaussian beam illuminates one end of the nanowires. According to recent experimental studies, the authors show that the propagation length of the localized surface plasmon excitations depends on the crystalline structure of the nanowire. Thus, they are able to determine the effective permittivity of metals in such a nanostructure versus its crystalline properties. The authors also demonstrate that the field of optical information transport could greatly benefit from the care of the subwavelength optical waveguide's crystallinity. (c) 2007 American Institute of Physics.
\end{abstract}

[DOI: 10.1063/1.2784389]

The employment of metallic materials is a well-known method to brake the diffraction limit in subwavelength optical waveguides. ${ }^{1}$ Indeed, the coupling of the incident wave to electron gas in metal allows the propagation of surface plasmon polariton (SPP) modes along the metal-surrounding medium interface. These modes decay exponentially along the directions perpendicular to the interface, and are thus highly confined to the surface. Krenn et al. experimentally investigated such nanostructures. ${ }^{2}$ A gold nanowire realized by a top-down technique was locally excited by a focused laser beam. An apertureless scanning near-field optical microscope (SNOM) was used to image the SPP propagation along the nanowire. Even if the SPP propagation length was rather small, the intensity map above the nanowire showed that this kind of nanostructures provides a good means to transfer optical information in nanoscale spaces and to locally address individual molecules or particles. Exploiting a similar configuration, Imura et al. experimentally studied a gold nanowire by dint of an aperture SNOM. ${ }^{3}$ Their intensity maps featured oscillating patterns reproduced by calculated maps. This phenomena corroborates the presence of SPP's propagation.

Using a bottom-up technique to realize a monocrystalline silver nanowire, Ditlbacher et al. showed that the propagation length of SPP highly increases. ${ }^{4}$ Indeed, there are less structural defects inducing plasmon damping in such a nanostructure than in the previous configuration realized with a top-down technique, and the silver nanowire behaves as a surface plasmon resonator. In a previously published work, we numerically confirmed these experimental results. ${ }^{5}$ However, the simulations have been developed by taking into account the usual tabulated permittivities. ${ }^{6-9}$ So, even if these numerical results are in fairly good agreement with the

\footnotetext{
${ }^{a}$ Electronic mail: thierry.laroche@utt.fr

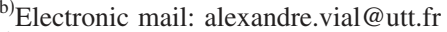

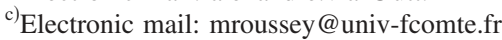

data reported in Ref. 4, they do not match the monocrystallinity of the experimental configuration. Indeed, we numerically found a SPP propagation length three times weaker than the experimental one. In a recent review article, ${ }^{10} \mathrm{Gi}-$ rard and Dujardin highlighted that the nanostructure's behavior under light excitation depends on their crystalline properties and also on the technique used for their realization. So, although the tabulated permittivities are commonly employed in most of the simulations about optical dispersive phenomena in nanostructures, they should not be used for all cases. Thus, we have to take into account the fabrication process for nano-optic simulations. In fact, each process provides a peculiar crystalline structure that leads to a specific optical dispersive behavior.

In this article, we numerically demonstrate the matter of the nanostructure's crystallinity. We specifically develop an efficient theoretical method to match as well as possible the experimental results for a monocrystalline nanostructure. Further, this method enables us to determine the real permittivity of the nanostructured material. The considered structure is depicted in Fig. 1. It consists in a monocrystalline silver nanowire of diameter $\Phi=120 \mathrm{~nm}$ smaller than the incident wavelength locally illuminated at one of its ends by a

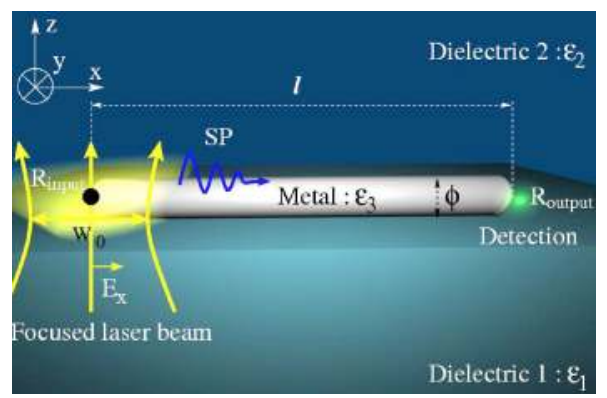

FIG. 1. (Color online) Perspective view of the local illumination by a focused Gaussian beam just beneath one end of a cylindrical metallic nanowire. 
TABLE I. Refection coefficient, propagation length of surface plasmon in a single silver nanowire, and permittivity for several values of $\kappa$ at $\lambda=785 \mathrm{~nm}$. The experimental column summarizes the values found in Ref. 4.

\begin{tabular}{cccccc}
\hline \hline$\kappa$ & 1 & 0.5 & 0.25 & Experimental & 0.34 \\
\hline$R$ & 0.180 & 0.205 & 0.201 & 0.216 & 0.204 \\
$L(\mu \mathrm{m})$ & 5.17 & 8.57 & 12.8 & 10.1 & 11.0 \\
$\epsilon$ & $-26.71+1.50 i$ & $-26.54+0.82 i$ & $-26.48+0.42 i$ & $\cdots$ & $-26.50+0.57 i$ \\
\hline \hline
\end{tabular}

focused Gaussian beam. The beam waist is set to $w_{O}$ $=1.5 \mu \mathrm{m}$ and the electric field polarization is along the main axis of the nanowire. The wire lies on a glass substrate $\left(\varepsilon_{1}\right.$ $=2.25)$ and is surrounded by air $\left(\varepsilon_{2}=1\right)$. The propagation conditions are numerically determined at the other end of the nanowire from a finite difference time domain (FDTD) method.

The FDTD method is a purely numerical scheme, applied to different near-field optical problems. ${ }^{11-13}$ The technique directly solves the time-dependent Maxwell equation using the central difference approximation in space and time domains meshed according to the scheme of Yee and Taflove and Hagness. ${ }^{14,15}$ Thus, both metallic and dielectric media are optically described by a three-dimensional grid built from their permittivities. We have applied this method to the geometry of Fig. 1 by using a three dimensional (3D)homemade FDTD code. In order to minimize the computation's time, we have implemented a $3 \mathrm{D}$ nonuniform grid with small space steps $\delta_{x}=10 \mathrm{~nm}$ and $\delta_{y}=\delta_{z}=5 \mathrm{~nm}$ to describe the nanowire. Elsewhere, the space step is $\Delta=20 \mathrm{~nm}$. This method allows us to narrow down the cells' number in the grid. For instance, the cells' number along the $x$ direction is $N_{x}=1281$ for $12 \mu \mathrm{m}$ long nanowire and $N_{y}=N_{z}=101$. Moreover, in order to avoid the nonphysical reflections, we have surrounded the main grid with Berenger's perfectly matched layer. $^{16}$

In the case of plasmonic metals, typical dispersion laws used for FDTD simulations are the Drude and Lorentz dispersion models. ${ }^{17}$ In this study, to characterize the optical dielectric function $\epsilon_{3}(\omega)$ of the silver nanowire, we have used the following model:

$$
\epsilon_{3}(\omega)=\epsilon_{\infty}-\frac{\omega_{D}^{2}}{\omega\left(\omega+i \gamma_{D}\right)}-\frac{\Delta \epsilon \Omega_{L}^{2}}{\left(\omega^{2}-\Omega_{L}^{2}\right)+i \Gamma_{L} \omega},
$$

where $\epsilon_{\infty}=3.91, \omega_{D}=13420 \mathrm{THz}$, and $\gamma_{D}=84 \mathrm{THz}$ are the usual constants of the Drude model, $\Omega_{L}=6870 \mathrm{THz}$ and $\Gamma_{L}$ $=12340 \mathrm{THz}$ represent the frequency and the spectral width of the Lorentz oscillator, and $\Delta \epsilon=0.76$ characterizes its strength. In the present work, these parameters have been optimized to fit the experimental data found in Ref. 6.

A large part of the nano-optic community agrees that the permittivity of nanostructured objects dissent from the bulk values. In metallic nanostructures, the mean free path of the electrons, related to the damping coefficients $\gamma_{D}$ and $\Gamma_{L}{ }^{18}$ is modified when the size of the structure decreases. This phenomena should increase the damping inside the nanostructure and thus change the permittivity.

In our case, the silver nanowire depicted in Fig. 1 is a monocrystalline nanostructure excited along its long axis. So, the previous argument does not work here. Indeed, the mean free path of electrons increases because of the absence of the crystalline domain walls. However, the approach is fairly similar to the one used for the damping phenomena. ${ }^{18}$ Instead of modifying the damping of the Drude and Lorentz models to decrease the mean free path of the electrons, we increase it.

This can be achieved for the silver nanowire by multiplying the previous Drude-Lorentz constants $\gamma_{D}$ ans $\Gamma_{L}$, respectively, by two constants $\kappa_{D}$ and $\kappa_{L}$. Thus Eq. (1) becomes

$$
\epsilon_{D L}(\omega)=\epsilon_{\infty}-\frac{\omega_{D}^{2}}{\omega\left(\omega+i \kappa_{D} \gamma_{D}\right)}-\frac{\Delta \epsilon \Omega_{L}^{2}}{\left(\omega^{2}-\Omega_{L}^{2}\right)+i \kappa_{L} \Gamma_{L} \omega} .
$$

To simplify the discussion, we consider as a first approach that $\kappa=\kappa_{D}=\kappa_{L}$.

In order to visualize the influence of the mean free path increase on the guiding efficiency of a single silver nanowire, we compare the near-field optical transmittance versus the incident wavelength when $\kappa$ decreases from $\kappa=1$ in Fig. 2 of Ref. 5 to $\kappa=0.25$ in Fig. 2 .

The spectra have been plotted for five wire lengths increasing from $l=4 \mu \mathrm{m}$ to $l=12 \mu \mathrm{m}$. The optical transmittance is defined in the same manner than in Ref. 5. If we compare the results in Fig. 2 of Ref. 5 and Fig. 2, respectively, for $\kappa=1$ and $\kappa=0.25$, it may be seen that the modulation depth is greater for $\kappa=0.25$ than the one found with $\kappa=1$. Moreover, the mean value of the transmittance is higher in the case of $\kappa=0.25$ whatever the length of the nanowire. So, it seems obvious that the electromagnetic transport is improved by increasing the mean free path of the electrons. Thus, this numerical method can be used to take into account the crystalline properties of a nanostructure. However, even if this method allows us to theoretically control the mean free path of the electrons in silver, we are not yet able to find the value of $\kappa$ which matches the permittivity of a single monocrystalline silver nanowire. To this end, we have to employ an empirical method. Otherwise said, we try

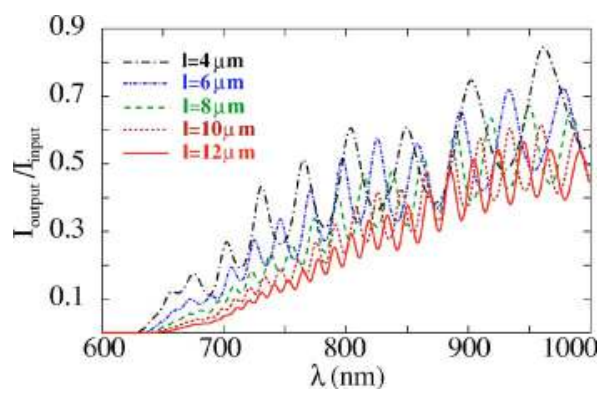

FIG. 2. (Color online) Normalized transmittance spectra computed for five different lengths of silver nanowires. The length increases from $l$ $=4 \mu \mathrm{m}$ to $l=12 \mu \mathrm{m}$. The wire diameter has been fixed to $120 \mathrm{~nm}$ and the polarization of the excitation electric field is parallel to the main axis of the nanowire (longitudinal mode). The dispersive aspect is taken into account with $\kappa=0.25$. 


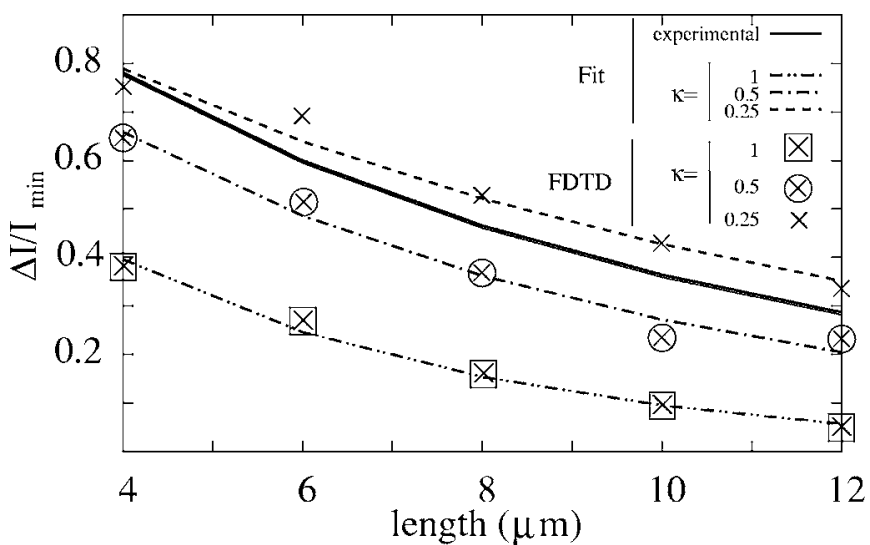

FIG. 3. Spectral relative modulation depth $\left(\Delta I / I_{\min }\right)$ at a wavelength equal to $785 \mathrm{~nm}$ for five different lengths of nanowire with diameter equal to $120 \mathrm{~nm}$.

to match experimental results obtained in Ref. 4 by varying the $\kappa$ coefficient in our numerical computation.

In a previous work, ${ }^{5}$ we highlighted the difference between theoretical and experimental propagation lengths of the surface plasmon mode $L$ when $\kappa=1$. We show in Fig. 3 the spectral modulation depths (crosses) and the associated Fabry-Perot cavity's fit (dashed lines) for several values of $\kappa$ and $l$. The way to measure these values is shown in Ref. 5. It may be seen in Fig. 3 that the mean value of the spectral relative modulation depth increases when the value of $\kappa$ decreases. Moreover, the calculation of the different fits, summarized in Table I, shows that the reflectivity and the propagation length of the surface plasmon increase too. Using a polynomial interpolation, we have found that the value $\kappa$ $=0.34$ minimizes the error between experimental and theoretical results. Corresponding values of $R$ and $L$ are 0.204 and $11.0 \mu \mathrm{m}$, respectively. This result allows us to define an adapted permittivity for the single monocrystalline silver nanowire, which is given by Eq. (2) for a fixed wavelength of $\lambda=785 \mathrm{~nm}$. Values of the permittivity for different $\kappa$ are given in Table I.
In this work, we have adapted the common tabulated permittivity to simulate a specific experimentation. We demonstrated a numerical method leading to a better correlation between experimental and theoretical results and we are now able to describe the propagation of surface plasmons on a monocrystalline metallic nanowire, in good agreement with the experiments. So far, we have used the same empirical parameter to modify both the Drude and the Lorentz parts of the permittivity. In further studies, different parameters could be employed for each term of the permittivity. The optimization of these parameters would require the use of specific methods such as evolutionary algorithms, already applied to recovering different kinds of nanostructure properties. ${ }^{19}$

${ }^{1}$ J. C. Weeber, A. Dereux, C. Girard, J. R. Krenn, and J. P. Goudonnet, Phys. Rev. B 60, 9061 (1999).

${ }^{2}$ J. R. Krenn, B. Lamprecht, H. Ditlbacher, G. Schider, M. Salerno, A. Leitner, and F. R. Aussenegg, Europhys. Lett. 60, 663 (2002).

${ }^{3}$ K. Imura, T. Nagahara, and H. Okamoto, J. Chem. Phys. 122, 154701 (2005).

${ }^{4}$ H. Ditlbacher, A. Hohenau, D. Wagner, U. Kreibig, M. Rogers, F. Hofer, F. R. Aussenegg, and J. R. Krenn, Phys. Rev. Lett. 95, 257403 (2005).

${ }^{5}$ T. Laroche and C. Girard, Appl. Phys. Lett. 89, 233119 (2006).

${ }^{6}$ Handbook of Optical Constants of Solids, edited by E. Palik (Academic, New York, 1985).

${ }^{7}$ Handbook of Optical Constants of Solids II, edited by E. Palik (Academic, New York, 1991).

${ }^{8}$ P. B. Johnson and R. W. Christy, Phys. Rev. B 6, 4370 (1972).

${ }^{9} \mathrm{M}$. Weber, Handbook of Optical Materials (CRC, London, 2003).

${ }^{10}$ C. Girard and E. Dujardin, J. Opt. A, Pure Appl. Opt. 8, S73 (2006).

${ }^{11}$ S. Maier, P. Kik, and H. Atwater, Phys. Rev. B 67, 205402 (2003).

${ }^{12}$ T. Laroche, F. Baida, and D. Van Labeke, J. Opt. Soc. Am. B 22, 1045 (2005).

${ }^{13}$ M. Roussey, F. I. Baida, and M.-P. Bernal, J. Opt. Soc. Am. B 24 (2007).

${ }^{14}$ K. Yee, IEEE Trans. Antennas Propag. 14, 302 (1966).

${ }^{15}$ A. Taflove and S. C. Hagness, Computational Electrodynamics: The Finite Difference Time Domain Method (Artech House, London, 2000).

${ }^{16}$ J. P. Berenger, J. Comp. Physiol. 127, 363 (1996).

${ }^{17}$ A. Vial, A.-S. Grimault, D. Macias, D. Barchiesi, and M. Lamy de la Chapelle, Phys. Rev. B 71, 085416 (2005).

${ }^{18}$ Near-Field Optics and Surface Plasmon Polaritons, edited by S. Kawata (Springer, Berlin, 2001), Chap. 5, pp. 97-122.

${ }^{19}$ D. Macías, A. Vial, and D. Barchiesi, J. Opt. Soc. Am. A 21, 1465 (2004). 